\section{LA DÉCADA PRODIGIOSA DE REGINA LAMO: SU COMPROMISO CON EL COOPERATIVISMO OBRERO EN LA CATALUÑA DE LOS AÑOS VEINTE}

\author{
Raquel Osborne \\ Universidad Nacional de Educación a Distancia \\ ORCID iD: https://orcid.org/0000-0003-4227-6155 \\ rosborne@poli.uned.es
}

\section{THE PRODIGIOUS DECADE OF REGINA LAMO: HER COMMITMENT TO WORKER COOPERATIVISM IN THE CATALONIA OF THE TWENTIES}

Cómo citar este artículo/Citation: Osborne, R. (2020). La década prodigiosa de Regina Lamo: su compromiso con el cooperativismo obrero en la Cataluña de los años veinte. Arbor, 196 (796): a551. https://doi.org/10.3989/arbor.2020.796n2004

Recibido: 26 mayo 2018. Aceptado: 23 octubre 2019.

RESUMEN: Este trabajo pretende articular la construcción del personaje de Regina Lamo, hasta ahora presentado como subalterno a otras vidas que han alcanzado más relevancia histórica que ella. Entre otros activismos, Lamo se convirtió en líder de opinión y en creadora de escuela en el terreno del cooperativismo como forma más eficaz de lucha contra la explotación del movimiento obrero en las primeras décadas del siglo XX en España. La grave situación de conflicto social vivido en esas primeras décadas del siglo en el contexto de la modernización de España, y en particular en Cataluña, junto con el auge del movimiento obrero, provocó una fuerte represión de la principal fuerza, el anarcosindicalismo. Se abrió así un espacio para los mutualistas que buscaban un desgaste gradual y pacífico del capitalismo y que estaban a favor de la economía social, frente a los bakuninistas, proclives a la lucha militante, la huelga general y la insurrección. En este marco, Regina Lamo perfila su compromiso con una original propuesta de creación de Bancos Populares de Crédito que singularizó su figura y la convirtió en un personaje relevante, transgresora de los límites de género, que hoy tratamos de reivindicar.

PALABRAS CLAVE: Regina Lamo; cooperativismo; anarquismo; compromiso; transgresión.
Copyright: () 2020 CSIC. Este es un artículo de acceso abierto distribuido bajo los términos de la licencia de uso y distribución Creative Commons Reconocimiento 4.0 Internacional (CC BY 4.0).
ABSTRACT: This paper looks to shape the construction of the character Regina Lamo, until now presented as subordinate to other lives that have more historical relevance than hers. Among other types of activism, Lamo became an opinion leader and the creator of a school in the field of cooperativism as the most effective way to fight against the exploitation of the labour movement in the first decades of the twentieth century in Spain. The serious situation of social conflict experienced in the first decades of the century in the context of the modernization of Spain, and in particular in Catalonia, along with the rise of the labour movement, caused strong repression of the main force, anarcho-syndicalism. A space was thus opened for the mutualists, who were looking for a gradual and peaceful erosion of capitalism and were in favour of the social economy, in opposition to the Bakuninists, prone to militant struggle, the general strike and insurrection. In this context, Regina Lamo defined her commitment with an original proposal for the creation of Popular Credit Banks, which singled out her position, one that today we look to reclaim as a transgressor of gender boundaries.

KEYWORDS: Regina Lamo; cooperativism; anarchism; commitment; transgression. 


\section{INTRODUCCIÓN}

Este artículo aspira a armar parcialmente la construcción del personaje de Regina Lamo. Se trata de una figura que hemos conocido hasta ahora casi exclusivamente asociada a la vida de otras personas que han alcanzado más relevancia histórica que ella ${ }^{1}$ : Rosario de Acuña, su cuñada de hecho, maestra y amiga (Fernández Riera, 2009); su hija mayor, Carlota O'Neill, autora del excelente relato Una mujer en la guerra de España (2006) sobre su paso por las cárceles franquistas, donde aparece Regina madre; su nieta Lidia Falcón, hija de la segunda hija de Regina, Enriqueta, para quien Regina aparece en sus memorias como su abuelísima en los primeros once años de su vida (Falcón, 1979/1989) o Lluis Companys, con quien compartió militancias sindicalistas cuando este creó la Unió de Rabassaires de Cataluña (Pomés i Vives, 2000).

Macrino Fernández Riera, que es junto con José Bolado uno de los grandes recuperadores de Rosario de Acuña, y que por la amistad de esta con Regina Lamo se ha aproximado -de manera muy fidedigna- a la figura de esta última, se hacía eco en 2010 de un comentario de Lidia Falcón sobre el silenciamiento histórico de Regina Lamo:

¿Cómo puede ser posible que una mujer que «se dedicó, apasionadamente, al activismo sindical y cooperativista», a la lucha feminista -muy a pie de calle, al lado de las mujeres del pueblo, al estilo de Rosario de Acuña-, a la defensa de los más desfavorecidos y a la difusión de una nueva cultura que regenerara la vieja patria sea hoy tan desconocida para la inmensa mayoría de los españoles? (Fernández Riera, 2010, 23 de febrero).

Aparte de los autores citados que, aunque sea colateralmente, son los que mejor se han ocupado de Regina Lamo, por internet circulan unas glosas mínimas sobre su figura, que se van trasmitiendo de blog en blog, de un autor a otro, que aproximadamente señalan lo siguiente:

"Fue periodista, propagandista, feminista, anarquista, cooperativista, rapsoda. Escribió artículos de prensa y ensayo, así como poesía y obras de teatro. Además, difundió con pasión el control de natalidad y el derecho al aborto, la eugenesia, la eutanasia y el amor libre. En su faceta feminista, fue activista de los derechos de la mujer" (http://reginalamo.blogspot.com.es/).

Este relato, más o menos literal, acabó recientemente incorporado a wikipedia (https://es.wikipedia. org/wiki/Regina_de_Lamo), si bien estas afirmaciones no suelen ir acompañadas de datos concretos o de fuentes fidedignas.
Pero ¿cómo empezar a trabajar de forma sustantiva sobre una cuasi desconocida, aunque la sabíamos en boca de bastantes personas? ¿Por dónde empezar y sobre qué aspecto de su vida y obra? Decidí abordar la vertiente de su fuerte compromiso con el cooperativismo obrero, que destacaba como especialmente fructífera en el conjunto de su trayectoria. La década de 1920 aparecía como catalizadora de su activismo en este terreno. En la primera parte del presente artículo presentaré algunos rasgos de los orígenes y el contexto temporal y doctrinario del cooperativismo en Cataluña en las primeras décadas del siglo XX. En el siguiente apartado me centraré en el compromiso de Regina Lamo con el cooperativismo obrero, presentando los datos más significativos sobre su activismo en relación con su proyecto de los Bancos Populares de Crédito. Finalmente, analizaré el significado de la aportación de Lamo en el contexto de la posición de las mujeres y del cooperativismo catalán en la Cataluña de los años veinte del siglo pasado, quedando abiertas futuras investigaciones. Las fuentes empleadas han sido una combinación de fuentes primarias, secundarias y comunicaciones con sus descendientes.

\section{EL COOPERATIVISMO EN LA CATALUÑA DEL PRI- MER CUARTO DEL SIGLO XX}

Los cooperativistas catalanes de los años 20 reconocían la influencia de diversas corrientes del socialismo utópico del siglo anterior, así como de las de orientación anarquista en el pensamiento económico cooperativista. La aproximación a Fourier se hizo mediante el influyente análisis de Charles Gide en su acercamiento al socialismo utópico, y en concreto a Fourier como un visionario. Su falansterio podía ser reconocido como un antecedente de cooperativa de consumo y de producción a la vez, solidarias entre sí y formando parte de una Asociación Cooperativa Integral, a pesar de que las masas obreras optaron por el socialismo marxista (Coll Creixel, 1928, 6 de julio, p. 3). A su vez, el pensamiento de Proudhon contenía aspectos de mutualismo que en una sociedad sin estado irían en el sentido de satisfacer las necesidades de los trabajadores por medio de la cooperación voluntaria. Por otra parte, a pesar de las diferencias estratégicas entre el cooperativismo y el anarquismo, también se nutrieron de las tesis colectivistas de Bakunin en tanto que formas de asociación útiles para el proceso de emancipación del proletariado (Dalmau Torvà y Miró i Acedo, 2010, pp. 90-91). Por su parte el anarcocomunista Kropotkin manifestaba sus simpatías hacia el cooperativismo porque vincu- 
laba al productor con el consumidor, eliminando al intermediario en el comercio y la industria gracias a la organización de los consumidores, y al capitalista con la producción, gracias a la organización del crédito mutuo, posiciones en las que reconocía la influencia de Fourier y de Owen (Kropotkin, 1917, 15 junio). Otra aportación relevante para el cooperativismo catalán fue la del ya citado Charles Gide (El Homenaje de la Cooperación Internacional a Charles Gide, 1847-1927 (1927, 3 de junio)), profesor de economía francés que orientó buena parte de su magisterio al estudio de estructuras económicas de carácter colectivista, en concreto hacia el cooperativismo, contribuyendo a desarrollar los principales axiomas del cooperativismo internacional. Defendía una economía social, para lo que consideraba preciso "unos servicios organizados de forma asociativa, una organización de previsión en forma de mutua y una economía autogestionada de tipo cooperativo" (véase Lucena Giraldo. Gide, Charles (1847-1932)).

Algunas de estas enseñanzas calaron en el seno del movimiento obrero catalán como para poder hablar de una corriente cooperativista, truncada tras el Primer Congreso Obrero de 1870 en Barcelona. En él se enfrentaron los defensores del cooperativismo, de tendencia reformista y a favor de la economía social, y los bakuninistas, proclives a la creación de sociedades de resistencia y a favor de la confrontación directa (Pérez Baró, 1974). Con la Ley de Asociaciones de 1887 y el Código Civil de 1889 se organizaron en España las primeras cooperativas agrarias, entre ellas las cajas rurales o cooperativas de crédito basadas en los supuestos de Raiffeisen, específicas para la agricultura. Esta corriente de pensamiento fue difundida en España por Joaquín Díaz de Rábago, vinculado a la Sociedad Económica de Santiago de Compostela y cuya gran aportación fue la difusión en España de los modelos alemanes de cooperativismo de crédito, a cuyas publicaciones acudirían numerosos cooperativistas españoles del primer tercio del siglo XX para fundamentar sus propuestas. El otro modelo alemán, creado por Schulze e introducido en Italia por Luzzatti, se basaba en bancos de anticipos parecidos a una sociedad de crédito convencional pero que resultaron poco exitosos en el ámbito agrario. Al gallego Díaz de Rábago, y también al terrateniente murciano Nicolás Fontes, que finalmente se decantaron por el método Raiffeisen, les motivaba la necesidad de atajar la usura en el campo y de propiciar el acceso a la pequeña propiedad, que tanto abundaba en Murcia como en Galicia y cuyos arrendamientos eran a muy largo plazo. Ambos defendían un siste- ma de responsabilidad solidaria ilimitada de los asociados, lo que les diferenciaba del método Schulze, donde la responsabilidad era limitada. No obstante, defendieron con reparos la acción directa de los agricultores en la gestión del capital, previendo la participación de agentes sociales, véase el clero, como una forma de control de los agricultores, frenando así las luchas de clase con el objetivo de limitar la expansión de ideologías como el republicanismo, el socialismo o el anarquismo en el mundo rural, otro de los objetivos de su forma de entender el cooperativismo (Martínez Rodríguez y Martínez Soto, 2008, pp. 92-101; Pérez Baró, 1974, p. 37).

Las primeras leyes de sindicatos agrícolas se promulgan en 1906 y 1908 con la exención de ciertos impuestos, lo que favoreció la creación de numerosas cooperativas bajo el nombre de sindicatos agrícolas, siguiendo el modelo francés, y de cajas rurales autónomas, que llegaron a tener más de 50.000 socios en 1918, aunque habrá que esperar a 1931 para tener la primera ley específica de cooperativas, conocida como ley Largo Caballero (Martínez Rodríguez y Martínez Soto, 2008, pp. 92-101).

Con el fin de siglo, la pérdida de las colonias, además de la guerra de Marruecos, con la continua llamada de los varones de las clases populares a filas, se creó un clima de inestabilidad en el contexto de los gobiernos alternativos conservadores y liberales propiciados por la Restauración de 1878. En Barcelona se entró en un periodo de huelgas, bombas y represión que culminó con la huelga general de 1909, a la que siguió la Semana Trágica y una fuerte represión, en la que casi mil presos fueron condenados a muerte, entre ellos Ferrer i Guàrdia, impulsor de La Escuela Moderna. En la siguiente década se reorganizó el movimiento obrero, comenzando por la creación de la CNT en 1910. Sucesivas huelgas jalonaron la vida cotidiana de Barcelona: entre las más importantes destacan la huelga del textil de 1913 , la de 1916 contra la carestía de la vida y la huelga general revolucionaria de 1917 por el conflicto de los tranvías.

La neutralidad de España en la Primera Guerra Mundial la favoreció económicamente y favoreció, desde luego, a Cataluña. La burguesía se fortaleció y aumentó la inmigración desde regiones más pobres (Dalmau Torvà y Miró i Acedo, 2010, pp. 56-57). Pero el periodo que va de finales de la I Guerra Mundial al golpe de Primo de Rivera en 1923 se caracterizó en Barcelona por la crisis económica y por la violenta lucha político-sindical: en los años 20 , las huelgas pasaron a ser más frecuentes y violentas. Las 53.356 
huelgas que tuvieron lugar entre 1910 y 1917 Ilegaron a 142.710 en el quinquenio 1918-1923. La CNT pasó de 15.000 afiliados en 1915 a 427.000 en 1919, año en que convocó una huelga que consiguió la jornada laboral de ocho horas (Pradas Baena, 2003, pp. 37-39). Pero la patronal incumplió los acuerdos, respondió con un lockout y fomentó junto a las autoridades -el gobernador de Barcelona, Martínez Anidola represión de los sindicatos, apoyando métodos particularmente violentos, entre ellos el pistolerismo -caso de los Sindicatos Libres- para romper las huelgas y desorganizar a los trabajadores, incluido el asesinato selectivo de dirigentes sindicales, el más conocido el del anarcosindicalista Salvador Seguí, el Noi del Sucre, en 1923 (Pradas Baena, 2003). Uno de los resultados de este clima de agitación fue la aparición de toda una generación de luchadores que fueron la base de la enorme fuerza del sindicalismo revolucionario. Fue creciendo en paralelo una nutrida red de locales de reunión, con fines de organización, educativos, formativos y culturales, incluidos los Ateneos Libertarios. En suma, un tejido comunitario muy amplio y consistente, cuyas huellas perviven hoy en día en la sociedad catalana (ArnabatMata y Ferré-Trill, 2017, p. 388; Dalmau Torvà y Miró i Acedo, 2010, pp. 60-62).

La dificultad de plantear la lucha de clases exclusivamente en forma de confrontación violenta que transformara el orden establecido revitalizó el papel del cooperativismo con el fin de evitar la violencia y el derramamiento de sangre. En este contexto de radicalización se apostó por "el arma de combate de la economía". Se trataba de conseguir un sistema de producción, distribución e intercambio de la riqueza justos frente a la desigualdad radical planteada por el capitalismo (Dalmau Torvà y Miró i Acedo, 2010, p. 136). Aunque las cooperativas obreras comulgaban con los fines de las organizaciones proletarias, como acabar con la explotación del hombre por el hombre, pretendieron convertirse en una alternativa al radical enfrentamiento de clases. Desde su óptica solo la confrontación y las huelgas, estrategia del anarcosindicalismo, no eran suficientes para acabar con el capitalismo. En este sentido señala Bookchin: "como 'cooperativistas', los mutualistas pretendían un desgaste gradual y pacífico del capitalismo. Los anarquistas, por su parte, resaltaban la necesidad de la lucha militante, la huelga general y la insurrección", así que "el movimiento cooperativista, quizá más auténticamente proudhiano que anarquista, puso muchos obstáculos al desarrollo revolucionario del movimiento anarquista español" (Bookchin, 2001, pp. 44-45).

\section{EL COOPERATIVISMO DE REGINA LAMO}

En este contexto se mueve Regina Lamo, que se traslada a Barcelona junto con su marido, Enrique O'Neill, y las hijas, Carlota y Enriqueta, en la segunda década del siglo XX tras el fallecimiento de la tercera hija del matrimonio el 28 de julio de 1914 en Madrid². En relación con el deceso relata Juan Antonio Hormigón que "al morir la pequeña Regina, la familia se trasladó a Barcelona, cosa que debió suceder a mediados de la segunda década del siglo" (Hormigón, 1997, p. 17). Empadronados en Madrid en 1905 y 1910, no los encontramos en los padrones de Madrid y Barcelona en 1915, desconociendo las razones de esta omisión. Reaparecen los cuatro miembros de la familia en el padrón municipal de Barcelona en $1920^{3}$. De todos modos, una vez que conocemos la fecha del fallecimiento de Regina O'Neill sabemos que su traslado tuvo lugar, como pronto, después de 1914. Pero no tenemos que esperar a que el padrón municipal nos confirme su residencia en 1920 para conocer que se hallaban en Barcelona desde hacía tiempo: a comienzos de 1917 vemos emerger públicamente a Regina Lamo ofreciendo una lectura de poesía en el Ateneu Barcelonès ${ }^{4}$. Con todo, no será hasta abril de 1918 cuando los diarios La Vanguardia y El Diluvio anuncien una conferencia suya sobre cooperativismo en el Fomento del Trabajo Nacional ${ }^{5}$. Es el comienzo de su andadura pública en torno al cooperativismo, que florece en los años 20 en Barcelona en la que podríamos denominar su década prodigiosa. Regina se sumerge, se integra, se mimetiza con el tejido cultural y asociativo de Barcelona, como comprobaremos a continuación.

De hecho, nuestra protagonista alude a esta conferencia en un documento excepcional sobre su destacada trayectoria en el activismo cooperativista ${ }^{6}$. El 16 de abril de 1920 Regina Lamo de O'Neill presentaba desde Barcelona una solicitud a la Junta de Ampliación de Estudios (JAE) en la que pedía una pensión para estudiar durante un año "las entidades bancarias de Crédito obrero" en otros países europeos más avanzados en este tema, a saber, Suiza, Italia, Alemania y Escocia (http://archivojae.edaddeplata.org/jae_app). Regina defendía la virtualidad de los Bancos obreros "como primera fórmula de concordia entre los elementos patronales y asalariados" (cursiva nuestra). No consiguió la beca, como tampoco la obtuvieron otras destacadas contemporáneas suyas como Magda Donato y su hermana Margarita Nelken, Concha Méndez o María Teresa León (Mangini, 2001, pp. 87-88). En su expediente no se encuentra documento alguno que explique por qué se la denegaron, pero sí vemos reflejada su inquie- 
tud por la falta de respuesta varios meses después, hasta el punto de que el 18 de agosto escribe desde Gijón, donde pasa el verano junto a Rosario de Acuña, interesándose por la suerte de su solicitud.

De entre los méritos que incluye en su solicitud a la JAE menciona su pertenencia "como miembro único femenino a la entidad barcelonesa Sociedad de Estudios Económicos (SEE), previa presentación de la memoria reglamentaria, como demostración de suficiencia y capacidad en materias económico-sociales" (véase Solicitud Beca a la Junta de Ampliación de Estudios). La SEE fue fundada en 1907 en el período noucentista de la historia de Cataluña por un grupo de intelectuales decididos a intervenir activamente en la vida pública de Cataluña para lograr la modernización de la sociedad catalana y su homologación con Europa. Su primer presidente, el economista Guillem Graell, dejó el cargo de secretario general de la organización patronal Fomento del Trabajo Nacional para presidir la nueva Sociedad (http://www.lasocietat.cat/historia/).

En la presentación de su currículum la misma Regina apunta a la conferencia antes aludida de abril de 1918 como el comienzo a partir del cual desarrolla una "propaganda infatigable" a través de conferencias en entidades de orientación plural, en congresos sobre cooperativismo y con escritos en diversos medios de comunicación. Es de suponer que en fechas recientes a la impartición de la conferencia en Fomento tuviera lugar la presentación de la memoria reglamentaria que la convirtió en miembro de la SEE, memoria que no hemos podido consultar por no hallarse en los fondos de la Sede de dicha institución, pero que en cualquier caso nos la sitúa en la segunda mitad de la primera década del siglo XX en Barcelona, primero estudiando para presentar la citada memoria y, tras dicha acreditación, conferenciando en instituciones de carácter económico claves en Cataluña como Fomento del Trabajo Nacional y la propia SEE.

La formación de Regina, nacida en el año 1870, se hallaba muy alejada de un perfil económico. Según el principal testimonio con que contamos, el de la biznieta Lidia Falcón sobre la memoria familiar (Falcón, 1979/1989), los padres de Regina, residentes en Úbeda, eran librepensadores y ateos, siendo Anselmo Lamo sastre de profesión y masón por devoción -las mujeres a priori no tenían acceso a esta institución (Ortiz Albear, 2005)-. Pertenecientes a la mediana burguesía liberal y acomodada, el matrimonio decide emigrar en los años 80 a Madrid ${ }^{7}$, donde pensaban que tendrían más oportunidades para su negocio y esperaban encontrar un entorno más propicio a sus inquietudes culturales y políticas y una mejor educación para sus hijos. Para Carlos, el mayor, la carrera de derecho. Regina continuaría con la carrera de piano y solfeo, comenzada en úbeda. En un contexto en que solo se concebían profesiones femeninas asociadas al rol doméstico -maestras, institutrices o matronas(Flecha, 1996, p. 24), y en el que el piano era una enseñanza deseable para una señorita burguesa, el convertirlo en carrera más allá del divertimento parecía una buena elección, influida seguramente por las dotes y aplicación de Regina, que obtuvo un Segundo Premio en 1888 y un Primer Premio en 1889 en la Escuela Nacional de Música y Declamación (Fernández Riera, 2009, p. 145) ${ }^{8}$. Pero Regina poseía un amplio bagaje cultural adquirido, junto a los estudios de piano, en el culto hogar familiar, lo que le facilitaría su destacada inserción en el mundo del cooperativismo (Falcón, 1979/1989).

Su interés por la economía debió llegar después, en la década de 1910 en Barcelona, fecha en que en España las mujeres solo podían cursar estudios universitarios en las carreras de Farmacia, Medicina, Filosofía y Letras y excepcionalmente en Ciencias (Flecha, 1996, p. 227 y ss.), figurando matriculadas únicamente 21 mujeres -frente a 9.200 varones- en el curso 1910-1911 y 438 mujeres -frente a 21.029 varones- en 1915-1916 (Domínguez Cabrejas, 2008, p. 48). En ningún caso, pues, hubiera podido Regina tener algún título universitario de Economía a causa de la imposibilidad de obtención del mismo, dada la situación universitaria para las mujeres en los comienzos del siglo XX. De hecho, "En España hubo que esperar a 1943 para que la Facultad de Ciencias Políticas y Económicas de la Universidad Central incluyera por primera vez a una mujer en su orla" (véase Mardones, I. G. Economía en femenino). El autodidactismo se imponía, pues, y no debía ser un mérito menor una acreditación conseguida a través de la prestigiosa SEE que, junto a la Cátedra de Economía, financiada por Fomento del Trabajo Nacional, formaba parte del proyecto de instauración de una Universidad Catalana. Ello ayudaría a explicar la impartición de la conferencia inicial sobre "su proyecto de Banco de Crédito para obreras" en la que hemos señalado como muy reputada sede del Fomento del Trabajo Nacional. "La patronal más antigua de Europa", como se autopresenta actualmente dicha entidad, se había constituido en una referencia de la sociedad catalana y de su vida económica. En 1899 abandera la petición de un modelo de gestión administrativa descentralizada y un concierto económico para Cataluña (https://www. foment.com/es/sobre-nosotros/historia/). 
Por suerte para nosotros la noticia de prensa referida en la nota 5 informa del guión de la conferencia, que resume buena parte de los intereses y los contenidos del cooperativismo de Regina:

Exordio.- Feminismo relacionado con las instituciones de previsión y ahorro.- Bancos populares de Crédito en Inglaterra, Francia y Alemania.- Necesidad de los Bancos de Crédito para el elemento trabajador.- Conveniencia de ellos para los patronos.- Única solución por tales medios para conseguir la total regeneración de España.- Conclusión.

Dos años después, el 30 de marzo de 1920, la vemos presentando el tema precisamente en la Sociedad de Estudios Económicos con una conferencia que lleva por título "Necesidad social de los Bancos Populares de Crédito". El comentario resalta que fue "calurosamente ovacionada, tanto por la brillantez y documentación de su trabajo, como por ser la primera mujer que se consagra a los de esta índole" (cursiva nuestra) ${ }^{9}$.

Tras su presentación en sociedad ante las élites económicas de Cataluña, Lamo vuelve su mirada a los principales destinatarios de sus mensajes propagandistas "encaminados a llevar al elemento obrero a la creación de los Bancos Populares". Se dirige a "los Ateneos Obreros catalanes", instituciones autogestionadas con fines de elevar el nivel educativo, de formación profesional y cultural de jornaleros y artesanos, ateneos cifrados en unos 150 en Cataluña entre 1900 y 1930 por Pérez Solá (Monés i Pujol-Busquets, 2010, p. 111). Asociados a las culturas políticas hegemónicas -"el catalanismo republicano autodeterminista, el anarquismo y el emergente populismo"-, logran su mayoría de edad en el periodo 1901-1920, mientras que entre 1921 y 1936 consiguen lo que Arnabat-Mata y Ferré-Trill (2017) llaman "una expansión politizada" (p. 406).

El sindicalismo obrero gozó asimismo de la atención de nuestra protagonista, como lo representa su mención de "la Sociedad de cilindradores y estampadores" 'La Constancia', núcleo de los sindicatos catalanes" (cursiva nuestra) (véase Solicitud Beca a la Junta de Ampliación de Estudios) ${ }^{10}$. Los antecedentes de esta Sociedad residen, en un contexto de movilización anarquista, en una nueva forma de organización obrera que se crea en la Cataluña de comienzos del siglo XX con una orientación más sindicalista, lo que les permitía desmarcarse del viejo societarismo de oficio, y con unas posiciones más incluyentes de las mujeres (Enrech Molina, 2007). Dicha Sociedad permitirá en 1912 organizar el primer sindicato femenino del Arte Fabril y Textil de Barcelona, 'La Constancia', tras ha- ber facilitado el ingreso de mujeres y niños durante los años anteriores y haberse adherido a la CNT. Esta combinación permitió al año siguiente convocar una exitosa huelga del textil algodonero, en la que pararon más de 50.000 huelguistas.

Apenas un mes después de presentar la solicitud a la JAE, en que todo el trabajo propagandístico mencionado tiene lugar en Cataluña, Regina Lamo obtiene en Valencia su mayor éxito en el terreno del cooperativismo, la creación del primer Banco de Crédito Obrero en España. Previamente había hecho labor propagandística en Valencia, pues una iniciativa de esta envergadura no se improvisa, como nos revela La Vanguardia cuando en septiembre de 1919 refiere:

Ha salido con dirección a Valencia y Madrid la distinguida escritora doña Regina Lamo de O'Neill, a fin de organizar la implantación del primer Banco de Crédito Obrero en Valencia ${ }^{11}$.

Comprobamos efectivamente su arraigo en Valencia cuando en marzo de 1920, en el Congreso Regional de Cooperativas de Cataluña, celebrado en el Palacio de Bellas Artes de Barcelona, "aprobáronse diferentes ponencias y un proyecto de estatutos muy importante presentado por doña Regina Lamo, delegada de las Cooperativas de Valencia, para la creación de un Banco de Crédito Popular" (cursiva nuestra). ${ }^{12}$ Solo dos meses después tendrá lugar la inauguración de dicho Banco en Valencia.

Todos estos esfuerzos primero de estudio, luego de propaganda, y finalmente organizativos culminan el 13 de mayo de 1920. Ese día, en los viveros municipales y presidida por el alcalde Ricardo Samper, por entonces militante en el blasquista Partido de Unión Republicana Autonomista (PURA), tuvo lugar la inauguración en Valencia del primer Banco Obrero de España, el Banco de Crédito Popular y Cooperativo de Valencia "Institución Regina Lamo", constituido bajo los auspicios de la Federación Cooperativa de dicha región. En la portada de la edición de los estatutos del Banco aparece una foto de "Doña Regina Lamo, cultísima líder feminista, iniciadora del Banco y Presidenta perpetua del Consejo de Honor" (Villa Benayas, 2007), redactora de los Estatutos aprobados recientemente en Barcelona, entre cuyos fines, que se definen en el artículo 1.ㅇ, encontramos los siguientes:

a) Recoger el ahorro de los asociados y hacerlo reproductivo en beneficio común.

b) Realizar operaciones de crédito mutuo entre los mismos asociados. 
c) Practicar el seguro mutuo contra el paro forzoso en el trabajo y contra la enfermedad.

d) Constituir capitales hereditarios a favor de las familias de los afiliados que fallezcan.

e) La construcción de casas baratas para los socios.

Apenas un año después la memoria presentada en la Asamblea General el Banco de Crédito cuenta ya 4.000 afiliados entre socios individuales y pertenecientes a las cooperativas, empezando por la Federación Cooperativa Valenciana, y las ocho cooperativas locales, tanto de carácter urbano como rural (Villa Benayas, 2007).

Regina Lamo pasa ese verano de 1920 en Gijón con sus hijas Carlota y Enriqueta compartiendo casa con la escritora Rosario de Acuña, quien convivía desde hacía muchos años con Carlos Lamo, el hermano de Regina. Desde que se conocieron en Madrid a finales de los años 80 del siglo XIX, Regina, a la sazón de 18 años, establece una profunda amistad con quien considera una maestra, que además se convierte en su cuñada de hecho. El 13 de julio El Noroeste, periódico "democrático independiente de la villa de Gijón", se hace eco de la llegada de "doña Regina Lamo", a la que presenta como "la distinguida escritora y socióloga, fundadora del Banco de Crédito Popular de Valencia" que por su intensa labor intelectual y social había recibido "juicios muy laudatorios" en los diarios valencianos y barceloneses"13. Pocos días después, el 25 de julio aparece en dicho medio el primer artículo firmado por Regina Lamo de O’Neill (1920, 25 de julio) titulado "Finalidad social de los bancos populares de crédito" sobre las ventajas de los bancos populares de crédito. Ese mismo día se están concretando en Barcelona los acuerdos del ya mencionado Congreso Regional de Cooperativas de Cataluña, con la constitución de la Federación Regional de Cooperativas de Cataluña, de la cual forma parte nuestra protagonista (Pomés i Vives, 2000 , p. 579). Asimismo, en septiembre se cambiará el nombre del órgano de prensa de dicha Federación, El Cooperatista, por el de Acción Cooperatista, que recuperará el formato de 4 páginas bajo la dirección de Joan Coloma Chalmeta, manteniendo la periodicidad quincenal hasta 1925, en que pasaría a ser semanal (Dalmau Torvá y Miró Acedo, 2010, pp. 133-134).

Al año siguiente, en mayo de 1921, tiene lugar en Madrid el I Congreso Nacional de Cooperativas Obreras, en el que Regina ejerce nada menos que la Vicepresidencia. Participaron 120 delegados de múltiples organizaciones en representación de 400 cooperativas y 150.000 cooperativistas- (Villa Benayas, 2007, p. 92). Allí estuvo comisionada por la Federación Valenciana de Cooperativas para presentar una ponencia sobre los "Bancos de crédito populares y cooperativas. Sus relaciones con los almacenes generales" (Pomés i Vives, 2000, p.579), aprobada por unanimidad. En ella se habla del Banco, tipo Regina Lamo, haciendo una diferenciación clara con otras experiencias, tipo Raiffeisen, Schulze o Luzzatti (Villa Benayas, 2007, p. 92). Ello nos indica el conocimiento que de estos modelos tenía Regina, su intención de diferenciarse de los mismos y su ambiciosa meta de acuñar un modelo o una variación de los modelos cooperativistas dominantes hasta el punto de crear una "marca propia".

Con todo este bagaje Lamo publica el Breviario de Autoeducación Cooperatista ${ }^{14}$ (Lamo, 1923), que se convierte en un hito en su activismo propagandista, publicitado a través de la Biblioteca de "Acción Cooperatista". Dicha Biblioteca se constituyó con los textos considerados básicos por la Federación Cooperatista de Cataluña para la difusión del cooperativismo y para lograr convertirlo en una alternativa social. Entre los textos de nueve autores vendidos por Acción Cooperatista en 1927, cuatro años después de su publicación inicial, se encuentra el Breviario de Lamo, única autora $^{15}$. No solo esta revista orgánica del cooperativismo de izquierdas catalán se hizo eco a lo largo de los años del Breviario en repetidas ocasiones, sino también el popular diario barcelonés El Diluvio $^{16}$, del que fue colaboradora durante los años veinte Regina Lamo (Toll, 2016). No nos extraña, pues, que esta obra se convirtiera en "uno de los textos de cabecera del movimiento cooperatista de los años 20" (Dalmau Torvà y Miró i Acedo, 2009, p. 335, n. 203). En ella se utiliza un estilo didáctico de preguntas y respuestas, de apelaciones directas al lector y de reiteración sucesiva de los argumentos para así reforzarlos.

Lamo expone en el Breviario las virtudes del cooperativismo, que expresa la solidaridad obrera de forma complementaria a la acción de las grandes entidades que "se disputan la supremacía como conductoras de las muchedumbres proletarias [...]: el republicanismo, el socialismo, el sindicalismo y el anarquismo [...]. En esas cuatro disciplinas, cabe holgadamente [...] la doctrina cooperatista integral: es la esencia material y moral de todas ellas" (Lamo, 1923, pp. 5-6). La aceptación del cooperativismo por los asociados a estas organizaciones propiciará el paso del individualismo egoísta al colectivismo organizado para el consumo y la producción, única vía para la satisfacción de las necesidades, no solo materiales, sino morales y espirituales de la humanidad. Se aspira a lograr la justicia y la libertad para todos los trabajadores. 
En esta obra Lamo se dirige sobre todo al anarquismo, principal fuerza sindical en Cataluña a través de la CNT, que vimos había alcanzado en 1919 la cifra de 427.000 afiliados. El ideario anarquista:

"esculpe en el tiempo y en el espacio los prolegómenos de una humanidad nueva, perfecta -inteligente, bella y buena- y rechaza por sistema absurdo -absurdas son todas las negociaciones- el único procedimiento viable para conseguir esa perfectibilidad que sueña con ver entronizada" (Lamo, 1923, p. 10).

¿A qué procedimiento se refiere Lamo? A la solidaridad en el trabajo, que facilita la asistencia mutua y el apoyo en las necesidades materiales por medio de la cooperativización de las mismas. Se trata de garantizar las necesidades básicas de alimentación y los servicios de crédito del trabajador para cubrir los accidentes del trabajo, así como la ausencia de trabajo por huelgas y lockout, construyendo así "el parapeto de aguante". Pero además el cooperativismo transformará el sistema de trabajo y el concepto de propiedad, de la que formará parte el trabajador y sobre la que tendrá el control, siendo Proudhon el gurú al que alude Lamo en este escrito. Algo de esto recogían los estatutos de los Bancos de Crédito Popular, redactados como sabemos por la señora Lamo.

En 1925, en una conferencia en la Cooperativa obrera La Reforma, de Vendrell, titulada "El cooperatismo ante los problemas obreros", Lamo sigue insistiendo en lo que considera un "lamentable error" por parte del sindicalismo anarquista, al que critica por no aceptar el cooperativismo, a pesar de servirse "en varias ocasiones" de la ayuda "franca y sincera de las Cooperativas"17.

\section{CONCLUSIONES}

En este artículo hemos contribuido a dar forma a la figura desdibujada y desenfocada de Regina Lamo. Nos encontramos a una mujer con un fuerte compromiso de activismo en la causa del cooperativismo, el más relevante de toda su trayectoria y por el que debería pasar a la historia con el protagonismo que por tal tarea merece. Todas las informaciones disponibles apuntan al carácter precursor de su activismo, de forma que su mera presencia en espacios públicos tan masculinizados como el sindicalismo, máxime si iba asociado al terreno de la economía como en el caso del cooperativismo, apuntaba a una trasgresión de la ciudadanía "en femenino".

Antes y al mismo tiempo que ella vislumbramos otras mujeres activistas en el cooperativismo, como Micaela Chalmeta (1858-1951), socialista y feminista además de periodista, de larga trayectoria en el cooperativismo (Dalmau Torvà y Miró i Acedo, 2010, pp. 162-166), o Balbina Pi (1896-1973), obrera sindicalista afiliada a la CNT, activa ya en la huelga de 1913 y defensora de la organización de mujeres cooperativistas, que cuaja a comienzos de los años 30 (Pi, 1928, 20 de enero; Santamaría, 2018, 16 de septiembre). Pero a finales de la década de 1910 Regina elevó el listón siendo la primera mujer que combinó activismo cooperativista y conocimiento experto en materia económica, hasta el punto de que un periódico tan reputado como La Vanguardia se hizo eco con asiduidad de las actividades de nuestra protagonista. Pianista y profesora de música y canto de profesión, su ida de Madrid a la Barcelona a mediados de la convulsa primera década del siglo XX la hizo interesarse en la problemática obrera y sindical del momento.

Por aquellos años el anarquismo se había convertido en la principal fuerza en Cataluña que promovía la lucha obrera contra la explotación de los trabajadores. Lamo manifiesta su aprecio por la ideología anarquista porque preveía la utopía de una humanidad nueva, inteligente y bella, sin autoridad ni Dios, exenta de cualquiera de las otras formas utilizadas para la opresión de la clase obrera. Pero sentía un profundo desacuerdo con la estrategia del anarcosindicalismo de confrontación directa y rechazo de toda negociación, lo que consideraba un obstáculo para la consecución de la solidaridad en el trabajo, solo posible en su visión a través del cooperativismo de izquierdas, que ella misma representaba. Enfoque de gran tradición en Cataluña desde el siglo XIX, revive su posibilidad de alternativa social para el movimiento obrero -de tendencia republicana, socialista y sobre todo anarquista- tras el ambiente de extrema confrontación de clase de los primeros años del siglo XX en Cataluña, que impedía un fluido movimiento obrero, condenado con frecuencia a la clandestinidad. Considerando que "la representación del anarquismo no son las bombas de dinamita ni la finalidad y representación del cooperatismo son los garbanzos", Lamo aspira a una síntesis que supere la tensión entre anarcosindicalismo y cooperativismo: "ambas escuelas [...] resplandecerán administradas y mantenidas por el Cooperatismo Universal...", afirma en su Breviario (Lamo, 1923, p. 21).

En este contexto Regina se enfrasca en un periodo de formación autodidacta, refrendado con la presentación de una memoria en la principal institución económica de Cataluña, la SEE, a la que pasa a formar parte "como miembro único femenino". Por medio de este estudio se dota de las herramientas 
intelectuales para difundir "la doctrina cooperatista integral", en particular bajo la forma de los Bancos de Crédito Popular. Esta forma de solidaridad es presentada como la auto-organización económica obrera que complementaría la lucha protagonizada a través de los sindicatos de clase, significaría la "primera fórmula de concordia entre los elementos patronales y asalariados", y en definitiva constituiría la única solución "para conseguir la total regeneración de España", en consonancia con los males del fin de siglo y las soluciones intentadas desde múltiples ámbitos de la política y la cultura.

Su planteamiento quedará institucionalizado en Valencia, donde efectivamente se crea el primer Banco Obrero de Crédito, sin duda la iniciativa más destacada de su intensa actividad en el terreno del cooperativismo. Al igual que sus contemporáneos correligionarios en el cooperativismo, Regina, estudiosa del tema, se vería inspirada por las corrientes ideológicas que les precedieron en el siglo XIX. Fourier, Kropotkin y sobre todo Proudhon, al que algunos investigadores asocian al movimiento cooperativista en su enfrentamiento con los anarquistas (Bookchin, 2001), influyeron en su enfoque de un mutualismo que fomentara la propiedad colectiva -la posesión- de los medios de producción como forma de salir del estricto marco de la propiedad privada, propiciándose así, como se ha dicho, el paso del individualismo egoísta al colectivismo organizado para el consumo y la producción (Lamo, 1923). Inexcusable fue que conociera el trabajo de Charles Gide, el internacionalizador del cooperativismo de izquierdas en la segunda mitad del siglo XIX, y cuyos textos fueron también publicados por la biblioteca de Acción Cooperatista, la misma que publicó su Breviario. Gide sentó los principios de la economía social, base del planteamiento de Lamo.

El cooperativismo conservador, regido sobre todo por los supuestos del modelo alemán más exitoso en España, el Raiffeisen, difundidos en nuestro país en el siglo XIX y consultados por los cooperativistas del primer tercio del siglo XX, no podía escapar al conocimiento de nuestra protagonista. Inspirado por los loables motivos de atajar la usura en el campo y propiciar el acceso a la pequeña propiedad, representaba un intento patronal de tutelaje de la clase obrera a través del clero para contentar sus ansias de mejora a la vez que controlar su autonomía material e ideológica (Martínez Rodríguez y Martínez Soto, 2008). El objetivo de Lamo era exactamente el contrario: la emancipación de la clase trabajadora por medio del mutualismo y el crédito obrero que propiciaría el cooperativismo, herramienta transversal de todos los ismos conocidos del obrerismo de izquierdas. De ahí que se hablara del Banco tipo Regina Lamo, para diferenciarlo del cooperativismo conservador tutelado por la patronal y la Iglesia Católica. Por eso podemos afirmar que el éxito de la propuesta protagonizada por Regina Lamo tuvo que ver con "el desarrollo de un modelo cooperativista alternativo al que por entonces estaban impulsando algunos miembros destacados del catolicismo español" (Fernández Riera, 2009, p. 151, n. 93).

\section{A MODO DE CIERRE}

Hemos dado muestra de la infatigable tarea de Regina Lamo repartida en diversos lugares de la geografía española, sobre todo en Cataluña y Valencia, con algún apunte en Madrid y Gijón, cuando viajar no era lo que es ahora. Con todo, mientras hemos ido avanzando en este trabajo sobre el activismo cooperativista de Regina Lamo, hemos vislumbrado diversos campos de estudio imposibles de acometer en el presente artículo, tanto por la acotación temática y espacial del mismo, como por las diversas fuentes pendientes de investigación.

En el estricto campo del cooperativismo, quedan extensas áreas por explorar, a saber: en Cataluña hemos nombrado pero no agotado la amplitud de su militancia, y en particular hemos omitido por completo su participación desde los comienzos en la creación y desarrollo del sindicato La Unió de Rabbassaires, liderado por Lluis Companys, tanto en los mítines como impartiendo conferencias, así como en las asambleas y congresos de dicha organización (Falcón, 1979/1989; O’Neill, 1971; Pomés i Vives, 2000). Y más allá de Cataluña, la diversidad e intensidad de su activismo en Valencia, Madrid y Gijón apenas han sido mencionadas.

Hemos observado que su cooperativismo se halla atravesado por otros dos ejes centrales de su polimilitancia: la socialista y la feminista. Como socialista la hemos encontrado a lo largo de toda la década asociada orgánicamente de diversas maneras a la UGT y al PSOE (Dalmau Torvà y Miró i Acedo, 2010; Ezama Gil, 2013; Pomés i Vives, 2000). Y en cuanto a su feminismo aparece de forma continuada como representante de mujeres socialistas, cooperativistas y obreras -condiciones compatibles entre sí-. Por otra parte, desde los comienzos de su activismo manifiesta la imbricación del cooperativismo con los principios del feminismo, pues sin la inclusión de las mujeres en esta perspectiva no obtendría el sistema cooperativo la eficacia deseada. 
En suma, hemos comprobado su fervor y capacidad activista, atravesados por su firme compromiso con el proyecto de regeneración de España por medio de la mejora de las clases populares, que pasaba por el cooperativismo, además de por su socialismo y feminismo todavía por estudiar. Medios para difundir sus mensajes fueron las conferencias y el trabajo periodístico, que constituían asimismo una labor alimenticia en la medida de lo posible, con lo que podemos augurar que en los diversos terrenos de sus militancias quede mucho por descubrir de su periplo viajero, profesional y activista.

\section{AGRADECIMIENTOS}

Este artículo se enmarca en el proyecto Género, compromiso y transgresión en España, 1890-2016 (FEM2016-76675-P), financiado por el Ministerio de Economía y Competitividad.

Mi agradecimiento a Mar Masip, de la Fundació Roca Galès, por su eficaz ayuda en relación con las fuentes de esta investigación, así como a Carlota Leret O’Neill, Lidia Falcón O’Neill y Laura Salas Leret, nietas y biznieta de Regina Lamo (de O’Neill), por su amable disposición a facilitar información y documentos sobre Regina Lamo.

\section{NOTAS}

[1] La excepción que confirma la regla la vemos en los trabajos de Villa Benayas (2007) y Ezama Gil (2013), cuyo tema central es Regina Lamo. Para este artículo hemos consultado los siguientes archivos: Archivo de la Villa (Madrid), Arxiu Històric de la Ciutat de Barcelona, Arxiu Municipal Contemporani de Barcelona, Fundació Roca Galès (Barcelona) y Societat d'Estudis Econòmics (SEE) (Barcelona).

[2] Padrón de 1910, índice onomástico (1910-1915): Regina O'Neill Lamo (28/04/1911, Inscripción no 478, distrito 4), (28/07/1914, Inscripción no 795 , distrito 4). Archivo de la Villa (Madrid).

[3] Hemos consultado de forma presencial el padrón municipal del Archivo de la Villa (Madrid), y por correo-e el Arxiu Municipal Contemporani de Barcelona y no hemos encontrado rastros de la familia O'Neill Lamo en 1915. Sí hemos localizado sus datos en el índice del padrón de Barcelona del distrito IV de 1920 (volumen 1672 O-R): domicilio Valencia 260 1.1. O'Neill Acosta, Enrique; Lamo Jiménez, Regina; Lamo, Carlota, y Lamo, Enriqueta.

[4] Lectura de poesías de Doña Regina Lamo, 1 de febrero. Butlletí de l'Ateneu Barcelonès núm. 09 (gen.-març 1917), p. 392. Disponible en: https://arca.bnc. cat/arcabib_pro/ca/catalogo_imagenes/grupo.do?path=1009857.
[5] El Diluvio, 23 de abril de 1918, p. 7 y La Vanguardia, 23 de abril de 1918, p. 3.

[6] En ese documento Lamo sitúa esta conferencia en el mes de marzo, mientras que las referencias encontradas sobre la misma corresponden al mes de abril, como hemos visto, así que pensamos que debió ser un error de su memoria.

[7] Lidia Falcón fecha dicho traslado en 1876 porque, según ella, Regina tenía seis años y Carlos diez (Falcón, 1989, pp. 17-18). Sin embargo, según Fernández Riera, la llegada debió tener lugar a mediados de los años 80 , pues en julio de 1885 Carlos figura como matriculado en el Instituto San Isidro para continuar el bachillerato que había comenzado en 1878 en el provincial de Jaén (Fernández Riera, 2009, p. 145). Lo manifestado por Fernández Riera se corresponde con la edad cronológica de Carlos al empezar el Bachillerato, en coincidencia con su fecha de nacimiento en 1868 , solo dos años mayor que Regina y no cuatro, como indica Lidia Falcón.

[8] Algunos autores mencionan que el premio fue obtenido en el Real Conservatorio Superior de Música de Madrid, siendo que esta institución, creada en 1830, cambió su denominación en 1868 por la de Escuela Nacional de Música y Declamación, para volver a la original en 1900.

[9] La Vanguardia, 30 de marzo de 1920, p. 4.
[10] A pesar de que Regina habla de cilindradores y estampadores, comprobamos por las investigaciones de Enrech Molina que la denominación exacta es la que menciona este autor, a saber, la Sociedad de Cilindradores y Aprestadores (Enrech Molina, 2007, p.152). De nuevo, Lamo parece escribir de memoria.

[11] La Vanguardia, 26 septiembre 1919, p.

[12] El Diluvio. Diario político de avisos, noticias y decretos, 72, 21 marzo de 1920, p. 13.

[13] El Noroeste. Diario democrático independiente, 13 de julio de 1920, p. 1.

[14] Utilizamos los términos cooperatismo o cooperatista cuando aparecen así escritos en la fuente original, pues era la denominación empleada en la época. En el resto de las ocasiones se utilizan los términos cooperativismo o cooperativista, más acordes con el lenguaje actual.

[15] Anuncio publicitario publicado en Acción Cooperatista, 29 abril de 1927, p. 2. Entre esos textos se encuentra Las primeras nociones de Economía Políti$c a$, de Charles Gide.

[16] El Diluvio, 23 mayo 1923, p. 13.

[17] El Diluvio, 1 de enero de 1925, p. 30. 


\section{BIBLIOGRAFÍA}

Arnabat-Mata, R. y Ferré-Trill, X. (2017). Evolución histórica de los ateneos en Catalunya (1836-1936). Historia Contemporánea, 55, pp. 383-420. https:// doi.org/10.1387/hc.18090

Bookchin, M. (2001). Los anarquistas españoles. Los años heroicos 1868-1936. Valencia: Numa.

Coll Creixel, J. (1928, 6 de julio). Fourier, precursor de la Cooperación. Acción Cooperatista, pp. 1-7.

Dalmau Torvà, M. y Miró i Acedo, I. (2010). Les cooperatives obreres de Sants. Autogestió proletària en un barri de BarceIona (1870-1939). Barcelona: La Ciutat Invisible Edicions.

Domínguez Cabrejas, M. R. (2008). Cien años de libre acceso de las mujeres a la Universidad. Zaragoza: Universidad de Zaragoza.

El Homenaje de la Cooperación Internacional a Charles Gide, 1847-1927 (1927, 3 de junio). Acción Cooperatista, pp.1-2.

Enrech Molina, C. (2007). Género y sindicalismo en la industria textil (1836-1923). En Borderías Mondéjar, C. (ed.). Género y políticas del trabajo en la España contemporánea: 1836-1936. Barcelona: Icaria, pp. 127-162.

Ezama Gil, M. A. (2013). Edición y compromiso: Regina de Lamo y la colección "La Novela Blanca". En Arriaga Flórez, M., Bartolotta, S. y Martín Clavijo, M. (eds.). Ausencias. Escritoras en los márgenes de la cultura. Sevilla: Arcibel editores, pp. 398-415.

Falcón, L. (1979/1989). Los hijos de los vencidos. Barcelona: Pomaire.

Fernández Riera, M. (2009). Rosario de Acuña y Villanueva. Una heterodoxa de la España del Concordato. Gijón: Zahorí Ediciones.
Flecha, C. (1996). Las primeras universitarias en España (1872-1910). Madrid: Narcea.

Hormigón, J. A. (ed.) (1997). Circe y los cerdos. Cómo fue España encadenada. Los que no pudieron huir, de Carlota O'Neill. Madrid: Asociación de Directores de Escena de España.

Kropotkin, P. (1917, 15 junio). Una carta de Kropotkin. Cooperatismo, 56, pp. 161163.

Lamo, R. (1923). Breviario de Autoeducación Cooperatista. Barcelona: Biblioteca de Acción Cooperatista.

Lamo de O'Neill, R. (1920, 25 de julio). Finalidad social de los bancos populares de crédito. El Noroeste, 8398, p.3.

Mangini, S. (2001). Las modernas de Madrid: las grandes intelectuales españolas de la vanguardia. Madrid: Península.

Martínez Rodríguez, S. y Martínez Soto, A. P. (2008). Los pioneros del cooperativismo agrario de crédito español (18801920). CIRIEC - España. Revista de economía pública, social y cooperativa, 63, pp. 89-112.

Monés i Pujol-Busquets, J. (2010). Los ateneos obreros y la formación profesional en Cataluña. Participación Educati$v a$, extra 1 De la educación popular al aprendizaje a lo largo de la vida, pp. 108-126.

O’Neill, C. (1971). Los muertos también hablan. México: Populibros-LaPrensa.

O'Neill, C. (2006). Una mujer en la guerra de España. Madrid: Oberon.

Ortiz Albear, N. (2005). Las mujeres en la Masonería. Málaga: Universidad de Málaga.

Pérez Baró, A. (1974). Historia de la cooperación catalana. Barcelona: Síntesis.
Pi, B. (1928, 20 de enero). La mujer en la cooperativa. Acción Cooperatista, 246, pp. 1-4.

Pomés i Vives, J. (2000). La Unió de Rabassaires: Lluis Companys i el republicanisme, el cooperativisme i el sindicalisme pagès a la Catalunya dels anys vint. Barcelona: Publicacions de l'Abadia de Montserrat.

Pradas Baena, M. A. (2003). L'anarquisme $i$ les lluites socials a Barcelona, 19181923: la repressió obrera i la violència. Barcelona: Publicacions de I'Abadia de Montserrat.

Toll, G. (ed.) (2016). El Diluvio. Memorias de un diario republicano y federalista de Barcelona (1858-1939). Barcelona: Ediciones Carena.

Villa Benayas, R. (2007). Regina Lamo Jiménez, una mujer cooperativista. Cuadernos Mujer y cooperativismo, 9, pp. 90-94.

\section{Recursos en línea}

Fernández Riera, M. (2010, 23 de febrero). Unas palabras de Regina de Lamo en memoria de su "excelsa ascendiente» [en línea]. Disponible en https://rosariodea.wordpress.com/2010/02/23/46unas-palabras-de-regina-de-lamoen-memoria-de-su-\%C2\%ABexcelsaascendiente\%C2\%BB/

Lucena Giraldo, J. Gide, Charles (1847-1932). La web de las biografías [En línea]. Disponible en: http://www.mcnbiografias. com/app-bio/do/show?key=gide-charles

Mardones, I. G. Economía en femenino. Mujeres en red. El Periódico Feminista [En línea]. Disponible en: http://www.mujeresenred.net/spip.php?article1267

Santamaría, A. (2018, 16 de septiembre). Balbina Pi (1896-1973), sindicalista, oradora i actriu. [En línea]. Disponible en: https://www.isabadell.cat/sabadell/ historia/balbina-pi-1896-1973-sindicalista-oradora-i-actriu/ 